\title{
Separation of cognitive domains to improve prediction of progression from mild cognitive impairment to Alzheimer's disease
}

\author{
Suzanne B Hendrix ${ }^{* 1}$ and Kathleen A Welsh-Bohmer ${ }^{2}$ \\ See related research by Tatsuoka et al., http://alzres.com/content/5/2/14
}

\begin{abstract}
Addressing causes of heterogeneity in cognitive outcomes is becoming more critical as Alzheimer's disease (AD) research focuses on earlier disease. One of the causes of this heterogeneity may be that individuals with deficiencies in different cognitive domains may perform similarly on a neuropsychological (NP) test for very different reasons. Tatsuoka and colleagues have applied a Bayesian model in order to integrate knowledge about cognitive domains relevant to each NP test with the observed outcomes from the Alzheimer's Disease Neuroimaging Initiative (ADNI) mild cognitive impairment data. This approach resulted in better prediction of AD diagnosis than more traditional approaches.
\end{abstract}

\section{Introduction}

Alzheimer's disease (AD) research is beginning to focus on earlier stages of disease, prior to formal diagnosis of $\mathrm{AD}$ dementia. This focus on early symptomatic disease and populations with mild cognitive impairment (MCI) introduces issues of heterogeneity since patients at these earlier stages have not yet met the more standardizing criteria of an AD diagnosis. Understanding this heterogeneity is the first step in effectively planning studies for these early populations.

\section{Review}

One of the barriers to understanding the heterogeneity in these early populations is the difficulty in isolating cognitive domains for measurement. Most neuropsychological (NP) tests require a number of cognitive processes

*Correspondence: shendrix@pentaracorp.com

'Pentara Corporation, 2180 East Claybourne Avenue, Salt Lake City, UT 84109, USA Full list of author information is available at the end of the article and, therefore, measures of multiple domains. In the previous issue of Alzheimer's Research \& Therapy, Tatsuoka and colleagues [1] get around this problem by identifying the multiplicity of cognitive domains measured by each NP test and also the level of ability in that domain that is required for maximum performance on the test. They use this information in a Bayesian statistical model that is designed to separate out the effects of different cognitive functions on the NP outcomes. Based on NP data collected in MCI subjects in the Alzheimer's Disease Neuroimaging Initiative (ADNI) study, the approach yielded several potential combinations of cutoffs for baseline NP test scores and apolipoprotein E (APOE) status values which predict imminent risk of progression to $\mathrm{AD}$ over the course of a 24-month period.

Importantly, the authors also illustrate that more commonly applied methods, such as factor analysis and logistic regression, are not able to achieve the same level of success in predicting a diagnosis of $\mathrm{AD}$, presumably because the specific domains are not able to be separated with those models. Separation of specific domains also allows grouping of the specific combinations of deficits into diagnostic subgroups (for example, amnestic singledomain $\mathrm{MCI}$, amnestic multidomain $\mathrm{MCI}$, and nonamnestic multidomain $\mathrm{MCI}$ ) which then can be analyzed in terms of biomarkers and disease outcomes.

\section{Conclusions}

The ultimate validation of the method awaits replication in larger, more diverse MCI populations, as the authors acknowledge, although the method appears promising. Many different statistical approaches are currently being applied to clinical outcomes in order to identify patients who will progress to $\mathrm{AD}$ or patients who will have different decline rates over time. The results from these analyses can be used to help us identify a particular population and then a sensitive clinical outcome for study in a clinical trial that will result in improved power for detecting a difference. These results may also be 
helpful for identifying important stratification variables to include in a statistical model for analyzing the data from a clinical trial. Ultimately, these different statistical approaches will be evaluated by comparing their success in predicting conversion or maximizing decline rates in new populations.

\section{Abbreviations}

AD, Alzheimer's disease; $M C l$, mild cognitive impairment; NP,

neuropsychological.

\section{Competing interests}

SBH is president and owner of Pentara Corporation (Salt Lake City, UT, USA), a consulting firm that consults with several pharmaceutical companies and non-profit groups that are conducting clinical trials in AD. KAW-B declares that she has no competing interests.

\section{Author details}

'Pentara Corporation, 2180 East Claybourne Avenue, Salt Lake City, UT 84109, USA. ${ }^{2} J o s e p h$ \& Kathleen Bryan Alzheimer's Disease Research Center (Bryan ADRC), Duke University, 2200 West Main Street, Suite A200, Durham NC 27705, USA

Published: 15 May 2013

\section{Reference}

1. Tatsuoka C, Tseng H, Jaeger J, Varadi F, Smith MA, Yamada T, Smyth KA, Lerner AJ; The Alzheimer's Disease Neuroimaging Initiative: Modeling the heterogeneity in risk of progression to Alzheimer's disease across cognitive profiles in mild cognitive impairment. Alzheimers Res Ther 2013, 5:14.

\section{doi:10.1186/alzrt176}

Cite this article as: Hendrix SB, Welsh-Bohmer KA: Separation of cognitive domains to improve prediction of progression from mild cognitive impairment to Alzheimer's disease. Alzheimer's Research \& Therapy 2013, 5:22. 